\title{
Tacrolimus Induced Diabetes Presenting As Recurrent Pyelonephritis - A Rare Clinical Presentation
}

\author{
Vivek Mohanty, Ravi Kant, Aditya Sudan
}

\begin{abstract}
Tacrolimus is immunosuppressive agent used in a variety of skin disorders including vitiligo. Systemic Tacrolimus has been known to cause diabetes by inducing insulin resistance and increasing the activity and expression of glucose transporter, SGLT1 . In addition to this Tacrolimus has direct beta cell toxicity and subsequent insulin secretory defects. Although systemic absorption of tropical Tacrolimus is minimal but cases have been reported where tropical use of Tacrolimus has been associated with the blood levels above therapeutic range causing potential toxicity . Here we highlight a interesting case of a 22 year old female who was on treatment with Tacrolimus ointment for Segmental Vitiligo presented with features of pyelonephritis with past history of similar recurrent episodes and on further investigations was diagnosed to have New Onset Diabetes Mellitus with elevated serum Tacrolimus levels suggesting Tacrolimus induced beta cell dysfunction and insulin resistance thus making it an interesting and rare presentation of potential toxicity.
\end{abstract}

Index Terms - Tacrolimus, Diabetes Mellitus, Pyelonephritis, Vitiligo.

\section{INTRODUCTION}

Tacrolimus is a macrolide antibiotic used an immunosuppressive agent which acts by inhibiting calcineurin which is involved in production of IL-2, a molecule required for the production and development of $\mathrm{T}$ cells [1]. It has been effective in a variety of dermatological condition like atopic dermatitis, psoriasis, vitiligo, pyoderma gangrenosum, lichen planus [2]. Tropical tacrolimus has been found effective in vitiligo where it promotes pigmentation of depigmented areas possibly through affecting the local imbalance of cytokines profile which have found to have a role in vitiligo [3]. Most of the Tacrolimus related toxicity is associated with its prolonged systemic use and includes Hypertension, Hyperglycaemia, Nephrotoxicity, Tremor, systemic infection, seizures, Headache and closely corelates with its plasma levels [4]. Long term use of Tacrolimus has been known to cause Diabetes mellitus [5]. Tacrolimus induced toxicity is directly related to its plasma trough level. Systemic absorption of Tropical tacrolimus is studies have been demonstrated to be minimal [6]. Here we highlight a interesting case of a 22 year old female who was on treatment with Topical Tacrolimus $(0.1 \%)$ for vitiligo since last 5 years presented to our department with pain abdomen

Published on August 11, 2020.

Vivek Mohanty, All India Institute of Medical Sciences Rishikesh, India.

(corresponding e-mail: vivekmohanty.92@gmail.com)

Ravi Kant, All India Institute of Medical Sciences Rishikesh, India.

Aditya Sudan, All India Institute of Medical Sciences Rishikesh, India. and burning micturition was diagnosed to have Pyelonephritis. Her past history revealed she had multiple similar episodes since the last 6 months for which she had undergone multiple hospitalisations. Investigation revealed she was having Diabetes though she had no past and family history of Diabetes. Fasting C peptide levels were sent and were found to be normal. Her serum Tacrolimus levels was found to be above normal range $(5-15 \mathrm{ng} / \mathrm{ml})$. Thus, she was diagnosed to have Topical Tacrolimus induced New onset Diabetes Mellitus manifesting as recurrent pyelonephritis thus making it a rare and interesting presentation.

\section{CASE PRESEnTAtion}

A 24 year old female presented with chief complaints of abdominal pain since last 7 days associated with burning micturition, urinary urgency and frequency and cloudy urine. She was found to be hypotensive at presentation .Her general examination revealed presence of pallor and multiple white patches all over the body suggestive of generalized non segmental vitiligo (Fig. 1a, 1b). Abdominal examination revealed renal angle tenderness. Rest system examination were within normal limits. Her CBC showed normocytic normochromic anaemia with elevated Total leucocyte count with neutrophilic predominance. Her Kidney function test was suggestive of pre-renal Acute kidney. Urine routine microscopy showed full field of pus cells. The patient had 3 similar episodes in the last 1 year with hospitalization for these episodes. Her previous NCCT done 3 months ago was suggestive of Right pyelonephritis and she had features of obstruction at that time for which she underwent DJ stenting (Fig. 2). A provisional diagnosis of septic shock secondary to urinary tract infection was made. NCCT KUB showed Bilateral kidney pyelonephritis with right sided pyonephrosis (Fig 3a, 3b). Pig tail was inserted in the right kidney for drainage of the pyonephrosis. A review of the history from the patient attendant revealed these recurring symptoms have started after she started using Topical Tacrolimus ( $0.5 \%)$ for the vitiligo since last 5 year. Random blood sugar of the patient during routine investigation was found to be elevated and she was diagnosed to have diabetes on the basis of HB1AC levels which were high suggesting uncontrolled diabetes. Her Cpeptide levels were found to be normal thus ruling out Type 1 Diabetes mellitus. Serum Tacrolimus levels were found to be above the therapeutic range $(31.6 \mathrm{ng} / \mathrm{ml})$. Thus, she was diagnosed to have New onset Diabetes mellitus due to probable Tacrolimus induced beta cell toxicity leading to recurrent pyelonephritis. 


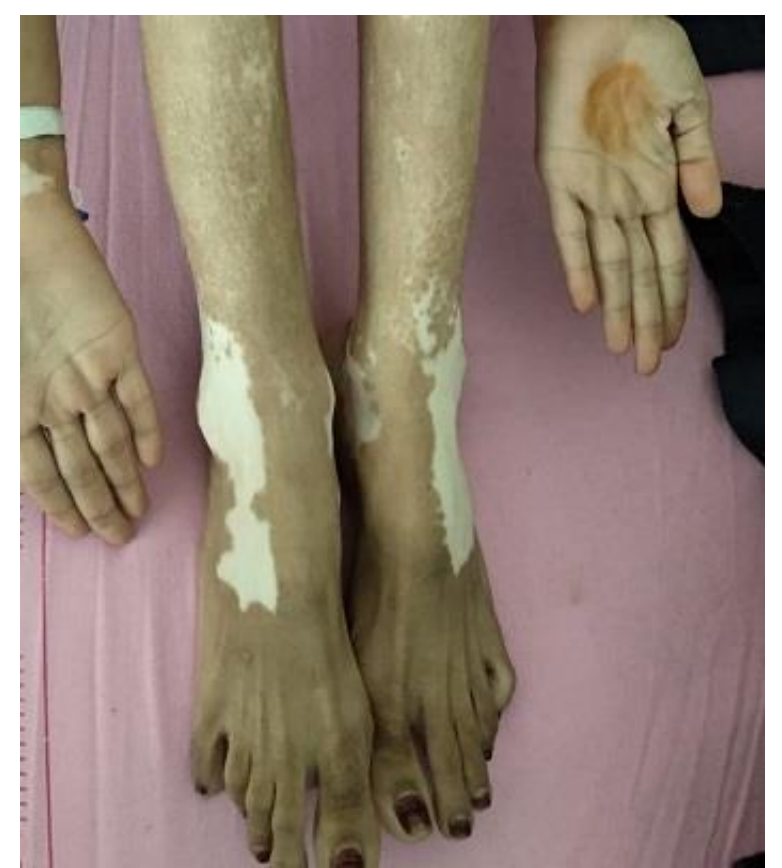

a)

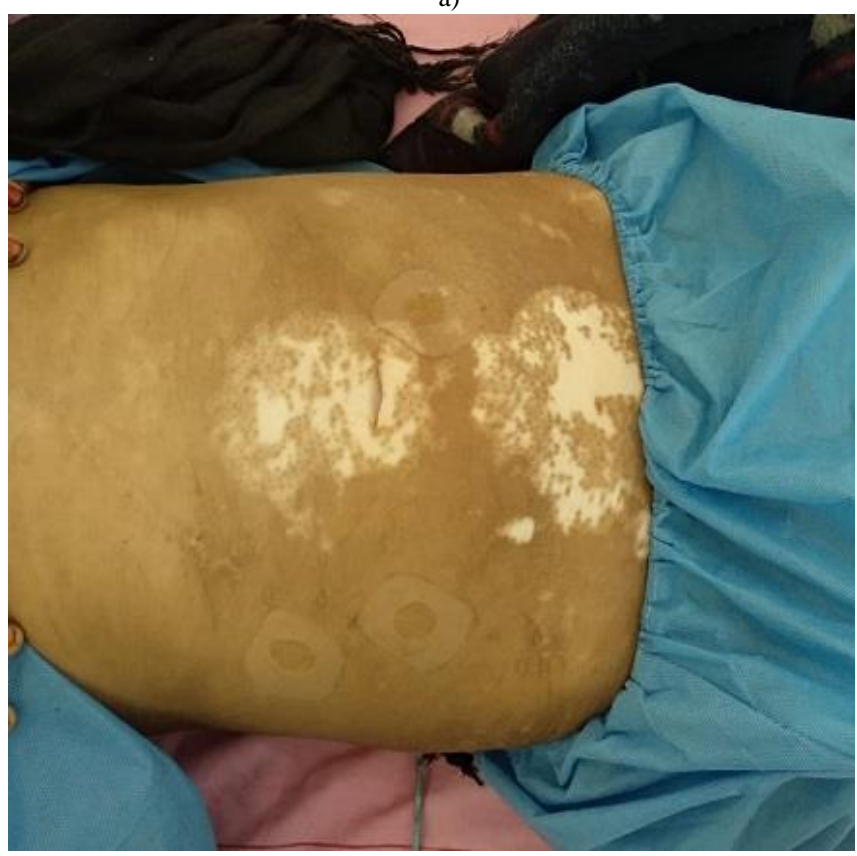

b)

Fig 1. a) Generalised Segmental Vitiligo involving bilateral feet; b) Generalised Segmental Vitiligo involving chest and upper abdomen.

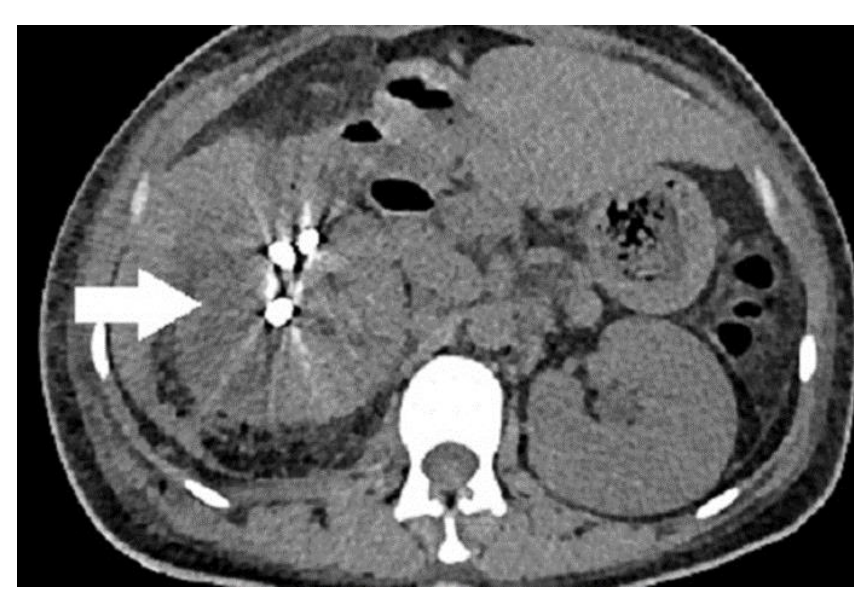

Fig. 2. Previous NCCT Abdomen axial view 3 month back showing right sided pyonephrosis with DJ stent in situ.
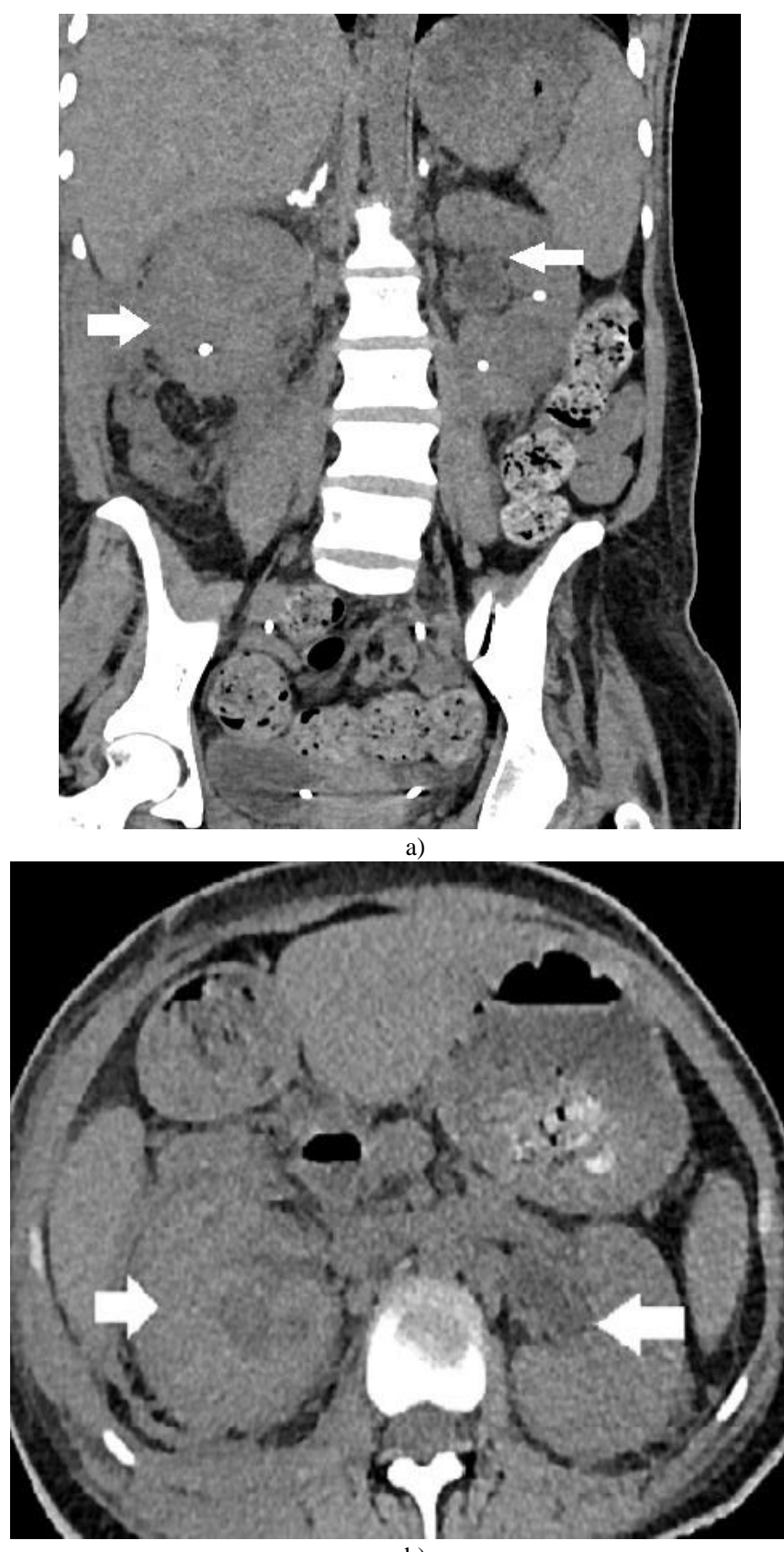

b)

Fig. 3. Recent NCCT abdomen coronal and axial view showing Left sided pyelonephritis with Right pyonephrosis

III. INVESTIGATIONS :

\begin{tabular}{|l|c|c|}
\hline$\underline{\text { Investigations }}$ & Day 1 & $\underline{\text { Day } 10}$ \\
\hline Haemoglobin (gm/dl) & $7.698 \mathrm{~g}$ & $7.32 \mathrm{~g} / \mathrm{dl}$ \\
\hline Total leucocyte count(cells/ $\mu \mathrm{l}$ & 24290 & $6789 / \mathrm{mm} 3$ \\
\hline $\begin{array}{l}\text { Differential count precentage } \\
\text { (N/L/E) }\end{array}$ & $86 / 5.3 / 4.8$ & $63 / 34 / 2.1$ \\
\hline Platelet count /mm3 & 385000 & 296000 \\
\hline $\begin{array}{l}\text { Blood urea / } \\
\text { Serum creatinine (mg/dl) }\end{array}$ & $125.6 / 3.02$ & $35 / 1.04$ \\
\hline Sodium/Potassium & $131 / 4.2$ & $132.4 / 4.8$ \\
\hline T. bilirubin/D. bilirubin (mg/dl) & $0.27 / 0.17$ & $0.37 / 0.19$ \\
\hline SGPT/SGOT (U/L) & $36.4 / 31.4$ & $47.8 / 41.2$ \\
\hline Alkaline phosphatase/GGU (U/L) & $280 / 48$ & $186 / 32$ \\
\hline Total protein/ albumin (gm/dl) & $7.02 / 3.92$ & $6.8 / 3.8$ \\
\hline HBA1C & $12.6 \%$ & \\
\hline Serum cortisol (microgram/dl) & 18.09 & \\
\hline TSH (mIU/ml)/FT4(ng/ml) & $0.95 / 1018$ & \\
\hline HBsAg/Anti HCV/Anti HIV & Non reactive & \\
\hline Fasting C-peptide levels (ng/ml) & 1.6 & \\
\hline
\end{tabular}


Urine Routine microscopy - pus cells full field.

Urine c/s - klebsiella sensitive to amikacin.

Pus c/s - proteus mirabilis sensitive to meropenem, gentamicin.

Serum Tacrolimus levels $-31.6 \mathrm{ng} / \mathrm{ml}$.

\section{TREATMENT AND FOLLOW UP}

The patient was resuscitated using fluids and started on inotropic support. Empirical antibiotics were added in the form of Fluroquinolones. She was also transfused with 1 units of Packed Red Blood cells. She was started on regular insulin for control of blood sugar. The patient became symptomatically better. Her antibiotic was changed according to urine and pus culture sensitivity report. Inotropes were gradually tapered and stopped. When the output from the pig tail became nil repeat. Repeat Kidney function test showed resolution of Acute kidney injury. Repeat NCCT KUB was done after 14 days of antibiotics which revealed no residual collection and pig tail was removed. Her Tacrolimus was discontinued. She was being discharged on low dose insulin and advised for regular follow up to look for potential reversibility of Diabetes upon stopping the drug.

\section{DISCUSSION}

Since absorption of tropical Tacrolimus is minimal so serum levels for potential toxicity are rarely achieved. However, cases have been reported where significant systemic absorption following tropical Tacrolimus application [7], [8]. Although no toxicities were demonstrated in these patients but the presence of significance drug levels in plasma may lead to potential toxicity. Such cases have mostly been seen following its application for treatment of peri-anal Crohns disease [9] and in cases of Graft versus Host disease [10] where the application of Tacrolimus resulted in severe uncontrolled Hypertension ,electrolyte abnormality and neurological symptoms. All these symptoms resolved after discontinuation of the drug.

Mechanism of Tacrolimus induced Diabetes mellitus includes decreased ATP production and glycolysis due to decreased glucokinase activity thus suppressing high glucose induced insulin secretion. Additionally, long term exposure can cause direct apoptosis and necrotic of the pancreatic beta islet cells [11]. Also, Tacrolimus affect the regulation of insulin gene transcription by a $\mathrm{Ca}^{2+}$ responsive pathway involving calcineurin and nuclear factor of activated $\mathrm{T}$ cells and may lead to decreased insulin secretion [12].

Our patient had developed diabetes secondary to Tacrolimus toxicity but was undiagnosed and was not any treatment for the same. Being immunocompromised she developed recurrent pyelonephritis and presented to us septicaemia and septic shock . Based on normal C-peptide levels , absence of family history of Type 2 diabetes mellitus with serum Tacrolimus levels above therapeutic range $(31.6 \mathrm{ng} / \mathrm{ml})$ she was diagnosed to have Drug induced Secondary Diabetes. This case highlights the importance of maintaining high degree of suspicion of systemic absorption and toxicity despite topical administration of immunosuppressants such as Tacrolimus despite such incidence being low. Here uncontrolled secondary diabetes due to Tacrolimus was not suspected or evaluated leading to recurrent pyelonephritis in a young patient without anatomical abnormality. Thus, patients with long term use of these drugs may required periodic testing for the same and modification of doses and temporary discontinuation of required. Special emphasis is to be given in case of dermatological and other disorders where the normal skin integrity may be disrupted leading to rapid systemic absorption of these drugs.

\section{CONCLUSION}

Thus, systemic absorption of topical Tacrolimus may be substantial with long term use in topical form and may manifest as drug toxicity in the form of Hyperglycaemia, HTN, Neurological manifestation and electrolyte imbalance. It should be kept as a provisional diagnosis in these patients after other causes have been ruled out. Treatment is mainly directed against discontinuation of the offending agent and regular follow up to look for potential reversibility.

\section{ACKNOWLEDGMENT}

Department of medicine and Radiology , AIIMS Rishikesh.

\section{FUNDING SOURCE}

This research did not receive any specific grant from funding agencies in the public, commercial, or not-for-profit sectors.

\section{REFERENCES}

[1] Thomson AW, Bonham CA, Zeevi A. Mode of action of tacrolimus (FK506): molecular and cellular mechanisms. Therapeutic drug monitoring. 1995 Dec 1;17(6):584-91.

[2] Cather JC, Abramovits W, Menter A. Cyclosporine and tacrolimus in dermatology. Dermatologic clinics. 2001 Jan 1;19(1):119-37.

[3] Grimes PE, Soriano T, Dytoc MT. Topical tacrolimus for repigmentation of vitiligo. Journal of the American Academy of Dermatology. 2002 Nov 1;47(5):789-91.

[4] Watson S, Pensabene L, Mitchell P, Bousvaros A. Outcomes and adverse events in children and young adults undergoing tacrolimus therapy for steroid-refractory colitis. Inflammatory bowel diseases. 2011 Jan 1;17(1):22-9.

[5] Penfornis A, Kury-Paulin S. Immunosuppressive drug-induced diabetes. Diabetes \& metabolism. 2006 Dec 1;32(5):539-46.

[6] Undre NA, Moloney FJ, Ahmadi S, Stevenson P, Murphy GM. Skin and systemic pharmacokinetics of tacrolimus following topical application of tacrolimus ointment in adults with moderate to severe atopic dermatitis. British Journal of Dermatology. 2009 Mar;160(3):665-9.

[7] Allen DM, Esterly NB. Significant systemic absorption of tacrolimus after topical application in a patient with lamellar ichthyosis. Archives of dermatology. 2002 Sep 1;138(9):1259-60.

[8] Allen A, Siegfried E, Silverman R, Williams ML, Elias PM, Szabo SK, Korman NJ. Significant absorption of topical tacrolimus in 3 patients with Netherton syndrome. Archives of dermatology. 2001 Jun 1;137(6):747-50.

[9] Ali FR, Lyon CC. Tacrolimus toxicity following topical treatment of perianal Crohn's disease: An admonitory anecdote. Journal of Crohn's and Colitis. 2013 Dec 15;7(12):e713

[10] Olson KA, West K, McCarthy PL. Toxic Tacrolimus Levels After Application of Topical Tacrolimus and Use of Occlusive Dressings in 
Two Bone Marrow Transplant Recipients with Cutaneous Graft-versus-Host Disease. Pharmacotherapy: The Journal of Human Pharmacology and Drug Therapy. 2014 Jun;34(6):e60-4.

[11] Radu RG, Fujimoto S, Mukai E, Takehiro M, Shimono D, Nabe K, Shimodahira M, Kominato R, Aramaki Y, Nishi Y, Funakoshi S. Tacrolimus suppresses glucose-induced insulin release from pancreatic islets by reducing glucokinase activity. American Journal of Physiology-Endocrinology and Metabolism. 2005 Feb;288(2):E365-71.

[12] Lawrence MC, Bhatt HS, Watterson JM, Easom RA. Regulation of insulin gene transcription by a $\mathrm{Ca} 2+$-responsive pathway involving calcineurin and nuclear factor of activated $\mathrm{T}$ cells. Molecular Endocrinology. 2001 Oct 1;15(10):1758-67. 